\title{
Dendritic Growth in Medial Prefrontal Cortex and Cognitive Flexibility Are Enhanced during the Postpartum Period
}

\author{
Benedetta Leuner and Elizabeth Gould \\ Princeton University, Department of Psychology, Neuroscience Institute, Princeton, New Jersey 08544
}

The postpartum period is associated with numerous hormonal changes, some of which have been linked to detrimental effects on the medial prefrontal cortex (mPFC), a brain region involved in cognition. Here, we investigated whether mPFC structure and function are negatively influenced during the postpartum period, an outcome that might be predicted by the maternal hormonal milieu. Mother rats were tested on an attentional set-shifting task, and dendritic architecture of layer 2/3 mPFC pyramidal neurons was analyzed. Mother rats exhibited increased dendritic spine number in the $\mathrm{mPFC}$, an effect that coincided with improved attention and behavioral flexibility. These findings suggest that the postpartum period is associated with promotion of neuronal growth in the mPFC and enhanced cognitive function.

\section{Introduction}

The postpartum period is accompanied by dramatic changes in hormones, including persistently increased glucocorticoid levels (Byrnes et al., 2007; Darnaudéry et al., 2007; Leuner et al., 2007; Pawluski et al., 2009). In virgin rats, high glucocorticoid levels have been associated with diminished dendritic architecture and dendritic spine density in the medial prefrontal cortex ( $\mathrm{mPFC}$ ) as well as concomitant decrements in cognitive tasks that require the mPFC (Liston et al., 2006; Radley et al., 2006). Elevated glucocorticoid levels have also been associated with heightened anxiety, the regulation of which has been linked to the PFC (Solomon and Herman, 2009).

Given the known detrimental effects of high glucocorticoid levels, it is perhaps surprising that new mothers with this hormonal profile exhibit attenuated stress reactivity, including reduced anxiety (Neumann, 2001; Macbeth and Luine, 2010). However, the influence of maternal experience on mPFC structure and cognitive function has not been investigated previously. One function of the mPFC is to modulate attentional processes and behavioral flexibility-lesions of the MPFC have been shown to impair performance on attentional set-shifting tasks (Birrell and Brown, 2000; Fox et al., 2003). In this task, animals are required to learn a series of associations between stimuli of certain sensory modalities and food rewards, and then switch these associations to other sensory modalities to effectively solve the task. Shifting attention from one sensory stimulus to another, referred to as an extradimensional shift (EDS), requires the mPFC (Birrell and Brown, 2000; Fox et al., 2003). Here, we examine whether mPFC pyramidal neurons and EDS performance are negatively

Received June 30, 2010; revised Aug. 6, 2010; accepted Aug. 12, 2010.

This work was supported by grants from the National Institute of Mental Health (MH84148 to B.L. and MH54970 to E.G.) and a Young Investigator Award from the National Alliance for Research on Schizophrenia and Depression, the Mental Health Research Association (B.L.). We thank E.R. Glasper for her assistance.

Correspondence should be addressed to Elizabeth Gould, Princeton University, Department of Psychology, Green Hall, Washington Road, Princeton, NJ 08544. E-mail: goulde@princeton.edu.

DOI:10.1523/JNEUROSCI.3388-10.2010

Copyright $\odot 2010$ the authors $\quad$ 0270-6474/10/3013499-05\$15.00/0 influenced during the postpartum period, outcomes that might be predicted by the maternal glucocorticoid profile. Contrary to our prediction, we found that the postpartum period is associated with enhanced neuronal growth in the $\mathrm{MPFC}$ as evidenced by an increased number of dendritic spines and that this structural change coincides with improved behavioral flexibility.

\section{Materials and Methods}

Animals. Age-matched adult cycling virgin and timed pregnant female Sprague Dawley rats from Taconic were used. Rats were housed individually on a $12 \mathrm{~h}$ light/dark cycle. On the day of birth [postpartum day 0 (PD0)], litters were culled to 10 pups. From PD0 to PD11, rats had access to food and water ad libitum, but thereafter were maintained on a restricted diet of $85 \%$ body weight. Water remained available ad libitum. All procedures were approved by the Princeton University Institutional Animal Care and Use Committee and conformed to the U.S. National Institutes of Health Guide for the Care and Use of Laboratory Animals.

Attentional set shifting. Virgin $(n=13)$ and mother $(n=13)$ rats were tested on an attentional set-shifting task (Fox et al., 2003; Liston et al., 2006) between PD20 and PD24 (Tables 1, 2). In the task, rats were trained to dig for a food reward (1/3 FrootLoop) and make discriminations based on a texture covering a digging container or a digging medium in which the reward was buried. The apparatus was an opaque Plexiglas box $(50 \times$ $40 \times 30 \mathrm{~cm})$ divided into three areas - a starting/holding area $(16 \times 40 \times$ $30 \mathrm{~cm}$ ) separated by a sliding opaque door from a choice area subdivided by a barrier into two $(34 \times 20 \times 30 \mathrm{~cm})$, each with a digging container (internal diameter and depth, $10 \mathrm{~cm}$ ). Textures covering the digging containers were made from various materials (Table 2). The attentional set-shifting procedure occurred over $3 \mathrm{~d}$. On each day, rats were brought to the testing room in their home cages and allowed to acclimate. After 10 min, rats were transferred to the starting area of the apparatus and the barrier lifted, allowing access to both containers. On the first day, each container was half filled with cob bedding and a reward was placed on top. After retrieving the reward from each container, rats were returned to the starting area and the containers rebaited. This procedure was repeated for three trials. If rats failed to retrieve both rewards within 5 $\mathrm{min}$, the trial was repeated. This habituation procedure was performed on the 10th to 13th day of food restriction. The next day, rats were shaped to dig for rewards buried within both containers and then trained on both a texture and medium simple discrimination (SD). Shaping was 
Table 1. Order of discriminations for the attentional set-shifting task

\begin{tabular}{|c|c|c|c|c|}
\hline \multirow[b]{2}{*}{ Discrimination } & \multicolumn{2}{|c|}{ Dimensions } & \multicolumn{2}{|c|}{ Exemplar combinations } \\
\hline & Relevant & Irrelevant & + & - \\
\hline SD & Medium & & M1 & M2 \\
\hline \multirow[t]{2}{*}{$C D$} & \multirow[t]{2}{*}{ Medium } & \multirow[t]{2}{*}{ Texture } & M1/T1 & $\mathrm{M} 2 / \mathrm{T} 2$ \\
\hline & & & $\mathrm{M} 1 / \mathrm{T} 2$ & $\mathrm{M} 2 / \mathrm{T} 1$ \\
\hline \multirow[t]{2}{*}{ IDS } & \multirow[t]{2}{*}{ Medium } & \multirow[t]{2}{*}{ Texture } & M3/T3 & M4/T4 \\
\hline & & & M3/T4 & $\mathrm{M} 4 / \mathrm{T} 3$ \\
\hline \multirow[t]{2}{*}{ REV } & \multirow[t]{2}{*}{ Medium } & \multirow[t]{2}{*}{ Texture } & M4/T3 & M3/T4 \\
\hline & & & M4/T4 & $\mathrm{M} 3 / \mathrm{T} 3$ \\
\hline \multirow[t]{2}{*}{ EDS } & \multirow[t]{2}{*}{ Texture } & \multirow[t]{2}{*}{ Medium } & T5/M5 & T6/M6 \\
\hline & & & T5/M6 & T6/M5 \\
\hline
\end{tabular}

$\overline{\text { Example of a typical sequence of discriminations, including the task-relevant dimension and all possible exemplar }}$ combinations. Half the rats switched from medium to texture and half switched from texture to medium. The correct exemplar is shown in bold for each discrimination and was paired with either exemplar from the irrelevant dimension. In the IDS and EDS, the stimuli were novel exemplars of each dimension. The order of presentation of exemplars and the assignment of exemplars into positive $(+)$ and negative $(-)$ stimuli were randomized in advance.

Table 2. Stimulus pairs

\begin{tabular}{ll}
\hline Medium pairs & Texture pairs \\
\hline Sawdust vs sand & Yellow fuzzy vs reversed yellow fuzzy \\
Gravel vs plastic beads & Waxed paper vs fine sandpaper \\
Ribbon vs crinkled paper strips & Velvet vs reversed velvet \\
\hline
\end{tabular}

The exemplars within a dimension (medium or texture) were always used in pairs. № two rats within a group received the same combinations, but groups of virgin and mother rats were matched.

done in four stages using containers filled with cob and the reward in each, as follows: (1) placed on top; (2) placed under a thin layer of cob; (3) buried beneath $\sim 2 \mathrm{~cm}$ of cob; and (4) buried under $\sim 4 \mathrm{~cm}$ of cob. The first three shaping stages consisted of three trials each. The last shaping stage consisted of six trials to ensure reliable digging. If rats failed to retrieve both rewards within $2 \mathrm{~min}$, the trial was repeated until they did so. Immediately after shaping, rats were trained to locate the reward based on either digging medium (crumpled tissue paper vs shredded latex gloves) or texture (short white fuzzy material vs reversed short white fuzzy material) to a criterion of six consecutive correct trials. The order of the SDs and positive stimuli were determined randomly and represented equally across rats. These stimuli were not used again. On the last day, rats were tested on a series of five discriminations (Tables 1,2). For all trials, rats had access to both containers, only one of which was baited with a reward. To eliminate the strategy of using odor to find the reward, all media included a small amount of powdered cereal. The left-right positioning of the baited container across trials was randomized. In the first four trials for each discrimination, rats could recover the reward even if it initially dug in the incorrect container, an error still being recorded. This was done to maintain responding on the task and to ensure sampling of both stimuli. After the first four trials, following an incorrect choice, rats were not permitted to recover the reward and were returned to the starting/holding area. Trials were terminated after $2 \mathrm{~min}$ if rats failed to approach a container and dig, and marked as an error. If five consecutive no-dig trials occurred, the test was terminated and continued on the following day. Testing started with the SD in which rats discriminated between either two textures or two digging media, one of which predicted the food reward (positive stimulus). Containers were filled with a neutral medium (cob) if the SD involved texture and were untextured if the SD involved medium. Next, in a compound discrimination (CD), a new dimension was introduced, but the positive stimulus was the same as in the SD. This was followed by an intradimensional attentional shift (IDS) involving two new exemplars from each stimulus dimension with the task-relevant dimension remaining the same as in the SD and CD. The IDS was then reversed (REV), such that the formerly negative stimulus became the positive stimulus. Finally, in the extradimensional attentional shift (EDS), two new exemplars from each dimension were introduced, and the formerly taskirrelevant dimension became relevant. Testing for each discrimination continued until a criterion of six consecutive correct responses was reached.
Golgi impregnation. Age-matched groups of mother (PD20-25; $n=7$ ) and virgin (diestrus $2 ; n=5$ ) rats were anesthetized with an overdose of sodium pentobarbital, and the brains were rapidly removed from the skull. Small blocks of tissue containing the PFC or hippocampus were immersed in a Golgi-Cox impregnation solution (Rapid GolgiStain kit, FD NeuroTechnologies), left undisturbed in the dark for 3 weeks, then transferred to $30 \%$ sucrose for $2 \mathrm{~d}$. Coronal sections $(125 \mu \mathrm{m})$ were cut on a Vibratome and stored in $15 \%$ sucrose in the dark at $4^{\circ} \mathrm{C}$. Before developing, all sections were mounted onto glass slides, placed in a humidity chamber in the dark, and dried overnight at $4^{\circ} \mathrm{C}$. For development, slides were incubated in 15\% ammonium hydroxide for $30 \mathrm{~min}$, rinsed in distilled water, incubated in undiluted Kodak photo fix for 30 min, rinsed, taken through a graded $(50,75,95$, and $100 \%)$ alcohol series ( $1 \mathrm{~min}$ each) with an extra $5 \mathrm{~min}$ rinse in $100 \%$ ethanol, cleared in Citrisolv for $5 \mathrm{~min}$, and coverslipped with Permount.

Microscopic analysis. All analyses were conducted blind to experimental conditions. Layer $2 / 3$ pyramidal neurons of the anterior cingulate area (Cg1-3) of the mPFC were analyzed. The Cg1-3 area is identified by its position on the medial wall of the rostral cortex and its location dorsal to infralimbic cortex, which is markedly thinner and has fewer, less welldefined cell layers (Paxinos, 1995; Wellman, 2001). Within Cg1-3, layer $2 / 3$ is recognized based on its positioning immediately ventral to the relatively cell sparse layer 1 and immediately dorsal to layer 4 , which has a smaller cell-packing density and larger somata size. mPFC pyramidal neurons were defined by their triangular soma, apical dendrites extending toward the pial surface, a basal dendritic tree, and dendritic spines. Neurons selected for analysis were fully impregnated with the soma located $150-450 \mathrm{~mm}$ from the pial surface. For comparison purposes, pyramidal neurons in layer $2 / 3$ of the orbitofrontal cortex (OFC), pyramidal cells in the ventral CA1 region and granule neurons of the dentate gyrus were also analyzed. On selected pyramidal neurons, five apical and five basal dendritic segments (each 10-25 $\mu \mathrm{m}$ long) were analyzed for dendritic spine density using a $100 \times$ oil objective on a BX-60 Olympus microscope equipped with a motorized stage and attached to a computer with StereoInvestigator software. On selected granule cells, five dendritic segments (each $10-25 \mu \mathrm{m}$ long) were analyzed similarly. For every cell, dendritic segments selected for analysis were as follows: (1) on secondary or tertiary dendrites; (2) $\geq 50 \mu \mathrm{m}$ away from the soma for apical dendrites and $30 \mu \mathrm{m}$ for basal dendrites; and (3) mostly in one focal plane. Only spines extending away from the shaft were counted. Randomly selected dendrites satisfying these criteria on three to five cells of each type per animal were examined. Due to variations in staining quality, 4-7 brains were analyzed for each cell type.

For dendritic length and branching analyses, cells were traced using the $40 \times$ objective providing an average length of apical and basal dendritic trees. Branch points were counted when a dendrite exhibited a bifurcation or juncture whereby two distinct branches were detected.

Radioimmunoassay. Age-matched groups of mother (PD7-10; $n=4$ ) and virgin (diestrus $2 ; n=4$ ) rats were anesthetized with an overdose of sodium pentobarbital between 12:00 and 2:00 P.M. Blood samples were obtained within 1-2 min by decapitation, added to test tubes containing $0.1 \mathrm{ml}$ of heparin, and centrifuged for $20 \mathrm{~min}$ at $3000 \mathrm{rpm}$. Plasma was collected and stored at $-20^{\circ} \mathrm{C}$. Circulating levels of corticosterone were measured using Coat-A-Count RIA kits (Diagnostic Products).

Statistics. Corticosterone levels and dendritic measures (dendritic spine density, branching, and length) were analyzed using two-tailed, unpaired Student's $t$ tests. For the attentional set-shifting task, trials to criteria (number of trials required to meet a criterion of six consecutive correct responses including the criterion run), and errors to criteria (number of errors made until animals reached a criterion of six consecutive correct responses) were analyzed using ANOVA with task phase (SD, CD, IDS, REV, EDS) as a within-subjects factor and reproductive experience (mother or virgin), and as a between-subjects factor. Bonferroni post hoc comparisons were applied when necessary.

\section{Results}

Corticosterone levels during the postpartum period

Consistent with previous reports showing elevated corticosterone levels throughout the postpartum period (Byrnes et al., 

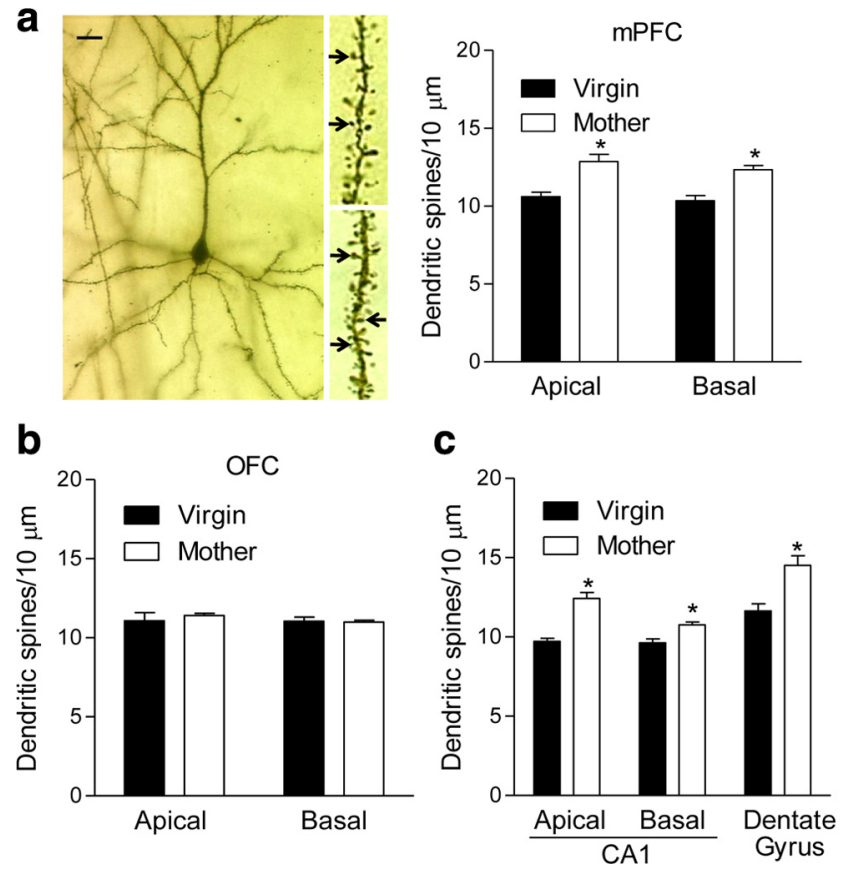

Figure 1. Mother rats exhibit neuronal growth in the MPFC and hippocampus. $\boldsymbol{a}$, Golgiimpregnated layer $2 / 3$ pyramidal neuron of the $\mathrm{mPFC}$, with magnified dendritic segments from virgin (top) and mother (bottom) rats. Arrows indicate dendritic spines. Mothers have more spines on apical and basal dendrites of $\mathrm{mPF}($ neurons. $\boldsymbol{b}$, Dendritic spines on layer $2 / 3$ pyramidal neurons of the $0 \mathrm{FC}$ were unaffected in postpartum rats. $c$, In the hippocampus, the postpartum period was also associated with increased dendritic spine density on pyramidal neurons of the ventral CA1 region and granule neurons of the dentate gyrus. Bars represent mean \pm SEM. ${ }^{*} p<0.05$. Scale bar: cell, $15 \mu \mathrm{m}$; segments, $5 \mu \mathrm{m}$.

Table 3. No change in dendritic length or branching was detected on mPFC pyramidal neurons during the postpartum period

\begin{tabular}{lllcl}
\hline mPFC & Apical length $(\mu \mathrm{m})$ & Basal length $(\mu \mathrm{m})$ & Apical branching & Basal branching \\
\hline Virgin & $1126.57 \pm 64.73$ & $1082.82 \pm 55.67$ & $9.93 \pm 0.59$ & $9.23 \pm 0.15$ \\
Mother & $1236.60 \pm 60.13$ & $1197.73 \pm 59.37$ & $10.63 \pm 0.65$ & $9.70 \pm 0.39$ \\
\hline
\end{tabular}

Dendritic length and dendritic branching for apical and basal dendritic trees of layer $2 / 3 \mathrm{mPFC}$ neurons in virgins and mothers. $p>0.05$ for all comparisons.

2007; Darnaudéry et al., 2007; Leuner et al., 2007; Pawluski et al., 2009), mother rats had elevated corticosterone levels compared with age-matched, cycling virgin female rats without reproductive experience (virgin: $124.4 \pm 55.48 \mathrm{ng} / \mathrm{ml}$; postpartum: $\left.293.4 \pm 43.03 \mathrm{ng} / \mathrm{ml} ; t_{(6)}=2.35, p<0.05\right)$.

\section{Dendritic spines in the MPFC and hippocampus during the postpartum period}

The postpartum period was associated with enhanced dendritic spine density on Golgi-impregnated pyramidal neurons located in layer $2 / 3$ of $\mathrm{mPFC}$ (Fig. 1a). Compared with virgin females, mothers had more dendritic spines on apical $\left(t_{(10)}=3.65, p<\right.$ $0.005)$ and basal $\left(t_{(10)}=4.75, p<0.001\right)$ dendrites. Neither dendritic length nor dendritic branching of mPFC pyramidal neurons was altered in postpartum rats (Table 3 ). Also, spine density on apical $\left(t_{(7)}=0.84, p=0.43\right)$ and basal $\left(t_{(7)}=0.23, p=\right.$ 0.82 ) dendrites of layer $2 / 3$ pyramidal neurons in the OFC did not differ between mothers and virgins (Fig. 1b). In the hippocampus, however, a brain region that projects to the $\mathrm{mPFC}$ (Verwer et al., 1997; Adhikari et al., 2010) and is known to be sensitive to maternal experience (Kinsley et al., 2006; Pawluski and Galea, 2006), ventral CA1 pyramidal cell apical $\left(t_{(6)}=6.49, p<0.005\right)$
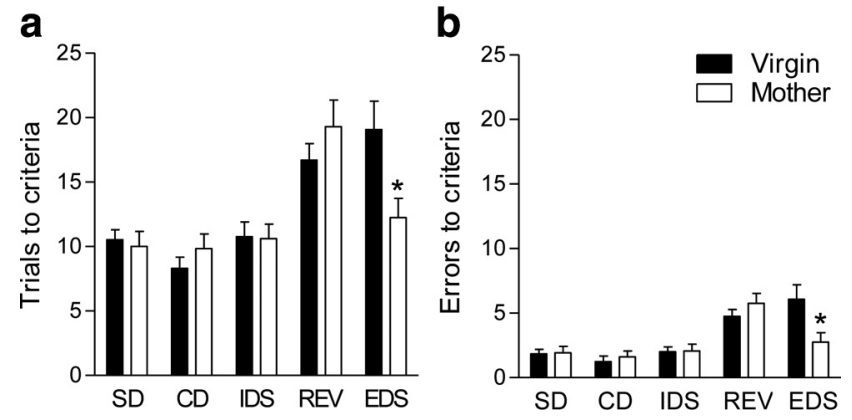

Figure 2. The postpartum period selectively enhances $\mathrm{mPFC}$-dependent cognitive function. Mothers and age-matched virgin females were tested on an attentional set-shifting task. $\boldsymbol{a}, \boldsymbol{b}$, Compared with virgins, mother showed enhanced extradimensional set shifting (EDS), as indicated by fewer trials $(\boldsymbol{a})$ and errors $(\boldsymbol{b})$ to reach criteria. Discrimination learning (SD, CD), intradimensional set shifting (IDS), and reversal learning (REV) were similar in virgins and mothers. Bars represent mean \pm SEM. ${ }^{*} p<0.05$.

and basal $\left(t_{(6)}=3.65, p<0.005\right)$ dendrites as well as granule cell $\left.t_{(7)}=3.05, p<0.05\right)$ dendrites exhibited a similar increase in spine density (Fig. 1c).

\section{Attentional set shifting during the postpartum period}

Mother rats showed a selective enhancement in extradimensional attentional set shifting, but not discrimination learning, intradimensional set shifting, or reversal learning (trials to criteria: $F_{(4,96)}=16.17, p<0.0001$; errors to criteria: $F_{(4,96)}=15.76, p<$ $0.0001)$. Mothers required fewer trials to reach criteria $(p<0.01)$ and made fewer errors $(p<0.01)$ relative to virgins on the EDS phase of the attentional set shifting task (Fig. 2). There were no significant differences between mothers and virgins in trials to criteria or errors to criteria on any other task phase. For both groups, more trials were required to reach criteria $\left(F_{(4,96)}=3.65\right.$, $p<0.001)$ and more errors $\left(F_{(4,96)}=3.82, p<0.01\right)$ were made during the REV and EDS phases than in the SD, CD, or IDS phase, thus confirming the greater difficulty of the REV and EDS tasks and the validity of the extradimensional attention shifting manipulation (Birrell and Brown, 2000; Fox et al., 2003; Liston et al., 2006). Since the REV and EDS tasks are equally difficult but mothers performed better only on the EDS and not the REV task, it is unlikely that increased caloric or metabolic demands of the mothers account for this pattern of results.

\section{Discussion}

Our results indicate that the postpartum period enhances dendritic spine density on pyramidal neurons of the mPFC and improves extradimensional set shifting, a task that depends on the mPFC (Birrell and Brown, 2000; Fox et al., 2003). These beneficial effects of the postpartum period occurred despite an adverse hormonal environment of elevated glucocorticoid levels, suggesting that the postpartum period not only buffers against hormonal changes but actually promotes neuronal growth in the mPFC while enhancing attention and behavioral flexibility.

The increase in dendritic spine density on mPFC pyramidal neurons occurred without a concomitant change in dendritic length or branching, indicating that changes in spine density reflect changes in the number of spines. Combined with studies showing that experimental manipulations increasing glucocorticoids, such as chronic aversive stress, decrease dendritic spine density of mPFC pyramidal neurons (Radley et al., 2006), it appears that dendritic spines on layer $2 / 3 \mathrm{mPFC}$ py- 
ramidal neurons rapidly respond to experience in order to meet the changing needs of the environment.

Diminished dendritic spine density and complexity of mPFC pyramidal neurons have been associated with impaired EDS performance in rats exposed to aversive stress or glucocorticoid injections (Wellman, 2001; Liston et al., 2006; Radley et al., 2006). Our findings are the first to show the converse-that increases in spine density are accompanied by improved performance on the EDS phase of the attentional set-shifting task. Thus, the postpartum period specifically improves cognitive functions associated with the $\mathrm{MPFC}$, suggesting that spine density changes may underlie improved cognitive ability. Consistent with this is the demonstration that the postpartum period did not affect reversal learning, which is dependent on the OFC (McAlonan and Brown, 2003), and did not produce any observable change in dendritic spines on OFC pyramidal neurons. Other work has similarly shown that stress-induced modifications in OFC dendritic arborization are not associated with reversal learning (Liston et al., 2006). Collectively, these results raise the possibility that functions mediated by the mPFC are more sensitive to experienceinduced structural changes.

Since dendritic spines are a primary site of excitatory synapses (Holtmaat and Svoboda, 2009), the postpartum period likely increased the overall number of excitatory synapses in the mPFC. Although the mechanism that controls this effect remains unknown, it is likely to be activity driven since dendritic spine synapse formation is triggered by synaptic activity (Nägerl et al., 2007). It is possible that neuronal activation within the PFC itself, which occurs with offspring contact (Fleming and Korsmit, 1996; Febo et al., 2005; Hernández-González et al., 2005), stimulates dendritic spine growth in the PFC. Another possibility is that the enhanced activity in the $\mathrm{mPFC}$ arises from the hippocampus. The ventral CA1 region of the hippocampus projects to the mPFC (Jay et al., 1989; Jay and Witter, 1991; Laroche et al., 2000), and this pathway shows evidence of synaptic plasticity (Verwer et al., 1997; Adhikari et al., 2010). Accordingly, dendritic spine increases in the dentate gyrus and CA fields of the hippocampus (Kinsley et al., 2006; Pawluski and Galea, 2006) during the postpartum period may augment activity in hippocampal afferents to the mPFC. This increase in activity may, in turn, lead to more dendritic spines and a related improvement in $\mathrm{mPFC}$ function. Future studies will be necessary to understand the relationship between structural plasticity in the hippocampus and mPFC, and how changes in these brain regions act individually or together to impact behavior.

Structural reorganization has also been shown to occur in the PFC of marmoset fathers. Unlike male rats, marmoset fathers care extensively for their offspring and will engage in the same behaviors as mothers, with the exception of nursing (FernandezDuque et al., 2009). Marmoset fathers exhibit enhanced dendritic spine density on layer 2/3 pyramidal neurons of the PFC (Kozorovitskiy et al., 2006). The PFC appears to be similarly altered in both sexes of two species with different social strategies, suggesting that this effect may be a phenomenon related to parenting in general. In this regard, it is worth noting that living in an enriched environment, in the absence of caregiving experience, is sufficient to induce comparable changes in dendritic spine density of pyramidal neurons in the PFC of marmosets (Kozorovitskiy et al., 2005). These findings suggest that the enriching aspect of parenting experience may be important for driving the changes in the mPFC of both mothers and fathers.

The postpartum period is a time of dramatic hormonal change. As shown here and previously (Byrnes et al., 2007; Dar- naudéry et al., 2007; Leuner et al., 2007; Pawluski et al., 2009), levels of glucocorticoids increase shortly after parturition. Our findings verified this hormonal profile relatively early in the postpartum period (postpartum days 7-10). Other studies have shown that corticosterone levels do not return to normal until around the time of weaning (Fischer et al., 1995; Byrnes et al., 2007). These findings raise the possibility that postpartum changes in brain structure and function may arise from persistent exposure to high corticosterone levels. Elevated corticosterone levels have been associated with diminished dendritic architecture and spine density in the mPFC and hippocampus (Sousa et al., 2000; Wellman, 2001; Listen et al., 2006; Radley et al., 2006; Hajszan et al., 2009). Reduced dendritic architecture and dendritic spines occur in association with decrements in cognitive tasks that require the $\mathrm{mPFC}$ and hippocampus (Sousa et al., 2000; Liston et al., 2006; Radley et al., 2006). Yet despite high corticosterone levels, we found enhanced dendritic spine density and improved cognitive flexibility during the postpartum period. These findings raise questions about protective factors associated with parenting that may buffer the $\mathrm{MPFC}$ from the adverse postpartum hormonal environment. In this regard, a role for oxytocin seems plausible as this neuropeptide is associated with the postpartum period and has been shown to protect the brain against some of the adverse effects of elevated glucocorticoids (Neumann, 2001; Windle et al., 2004).

Although lesions of the PFC do not have a profound effect on in the initiation of parenting behavior, some evidence in rodents suggests that pup retrieval may be affected (Afonso et al., 2007). The PFC has been implicated in many functions that may indirectly influence parenting, including attention, cognitive flexibility, and mood regulation (Dalley et al., 2004; Price and Drevets, 2010). Enhanced attentional abilities have been related to higher levels of maternal responsiveness (Lovic and Fleming, 2004). In human mothers, the PFC is responsive to stimuli associated with one's own offspring (Swain, 2008). Given that activity in this brain region is reduced with depression (Price and Drevets, 2010), it is possible that postpartum mental illness arises from an inability to engage mechanisms intended to protect the PFC from the negative consequences of the postpartum hormonal environment.

\section{References}

Adhikari A, Topiwala MA, Gordon JA (2010) Synchronized activity between the ventral hippocampus and the medial prefrontal cortex during anxiety. Neuron 65:257-269.

Afonso VM, Sison M, Lovic V, Fleming AS (2007) Medial prefrontal cortex lesions in the female rat affect sexual and maternal behavior and their sequential organization. Behav Neurosci 121:515-526.

Birrell JM, Brown VJ (2000) Medial frontal cortex mediates perceptual attentional set shifting in the rat. J Neurosci 20:4320-4324.

Byrnes EM, Bridges RS, Scanlan VF, Babb JA, Byrnes JJ (2007) Sensorimotor gating and dopamine function in postpartum rats. Neuropsychopharmacology 32:1021-1031.

Dalley JW, Cardinal RN, Robbins TW (2004) Prefrontal executive and cognitive functions in rodents: neural and neurochemical substrates. Neurosci Biobehav Rev 28:771-784.

Darnaudéry M, Perez-Martin M, Del Favero F, Gomez-Roldan C, GarciaSegura LM, Maccari S (2007) Early motherhood in rats is associated with a modification of hippocampal function. Psychoneuroendocrinology 32:803-812.

Febo M, Numan M, Ferris CF (2005) Functional magnetic resonance imaging shows oxytocin activates brain regions associated with mother-pup bonding during suckling. J Neurosci 25:11637-11644.

Fernandez-Duque E, Valeggia CR, Mendoza SP (2009) The biology of paternal care in human and non-human primates. Annu Rev Anthropol 38:115-130. 
Fischer D, Patchev VK, Hellbach S, Hassan AH, Almeida OF (1995) Lactation as a model for naturally reversible hypercorticalism plasticity in the mechanisms governing hypothalamo-pituitary-adrenocortical activity in rats. J Clin Invest 96:1208-1215.

Fleming AS, Korsmit M (1996) Plasticity in the maternal circuit: effects of maternal experience on Fos-Lir in hypothalamic, limbic, and cortical structures in the postpartum rat. Behav Neurosci 110:567-582.

Fox MT, Barense MD, Baxter MG (2003) Perceptual attentional set-shifting is impaired in rats with neurotoxic lesions of posterior parietal cortex. J Neurosci 23:676-681.

Hajszan T, Dow A, Warner-Schmidt JL, Szigeti-Buck K, Sallam NL, Parducz A, Leranth C, Duman RS (2009) Remodeling of hippocampal spine synapses in the rat learned helplessness model of depression. Biol Psychiatry 65:392-400.

Hernández-González M, Navarro-Meza M, Prieto-Beracoechea CA, Guevara MA (2005) Electrical activity of prefrontal cortex and ventral tegmental area during rat maternal behavior. Behav Processes 70:132-143.

Holtmaat A, Svoboda K (2009) Experience-dependent structural synaptic plasticity in the mammalian brain. Nat Rev Neurosci 10:647-658.

Jay TM, Glowinski J, Thierry AM (1989) Selectivity of the hippocampal projection to the prelimbic area of the prefrontal cortex in the rat. Brain Res 505:337-340.

Jay TM, Witter MP (1991) Distribution of hippocampal CA1 and subicular efferents in the prefrontal cortex of the rat studied by means of anterograde transport of Phaseolus vulgaris-leucoagglutinin. J Comp Neurol 313:574-586

Kinsley CH, Trainer R, Stafisso-Sandoz G, Quadros P, Marcus LK, Hearon C, Meyer EA, Hester N, Morgan M, Kozub FJ, Lambert KG (2006) Motherhood and the hormones of pregnancy modify concentrations of hippocampal neuronal dendritic spines. Horm Behav 49:131-142.

Kozorovitskiy Y, Gross CG, Kopil C, Battaglia L, McBreen M, Stranahan AM, Gould E (2005) Experience induces structural and biochemical changes in the adult primate brain. Proc Natl Acad Sci U S A 102:17478-17482.

Kozorovitskiy Y, Hughes M, Lee K, Gould E (2006) Fatherhood affects dendritic spines and vasopressin Vla receptors in the primate prefrontal cortex. Nat Neurosci 9:1094-1095.

Laroche S, Davis S, Jay TM (2000) Plasticity at hippocampal to prefrontal cortex synapses: dual roles in working memory and consolidation. Hippocampus 10:438-446.

Leuner B, Mirescu C, Noiman L, Gould E (2007) Maternal experience inhibits the production of immature neurons in the hippocampus during the postpartum period through elevations in adrenal steroids. Hippocampus 17:434-442.

Liston C, Miller MM, Goldwater DS, Radley JJ, Rocher AB, Hof PR, Morrison JH, McEwen BS (2006) Stress-induced alterations in prefrontal cortical dendritic morphology predict selective impairments in perceptual attentional set-shifting. J Neurosci 26:7870-7874.
Lovic V, Fleming AS (2004) Artificially-reared female rats show reduced prepulse inhibition and deficits in the attentional set shifting task-reversal of effects with maternal-like licking stimulation. Behav Brain Res 148:209-219.

Macbeth AH, Luine VN (2010) Changes in anxiety and cognition due to reproductive experience: a review of data from rodent and human mothers. Neurosci Biobehav Rev 34:452-467.

McAlonan K, Brown VJ (2003) Orbital prefrontal cortex mediates reversal learning and not attentional set shifting in the rat. Behav Brain Res 146:97-103.

Nägerl UV, Köstinger G, Anderson JC, Martin KA, Bonhoeffer T (2007) Protracted synaptogenesis after activity-dependent spinogenesis in hippocampal neurons. J Neurosci 27:8149-8156.

Neumann ID (2001) Alterations in behavioral and neuroendocrine stress coping strategies in pregnant, parturient and lactating rats. Prog Brain Res 133:143-152.

Pawluski JL, Galea LA (2006) Hippocampal morphology is differentially affected by reproductive experience in the mother. J Neurobiol 66:71-81.

Pawluski JL, Charlier TD, Lieblich SE, Hammond GL, Galea LA (2009) Reproductive experience alters corticosterone and CBG levels in the rat dam. Physiol Behav 96:108-114.

Paxinos G (1995) The rat nervous system. San Diego, CA: Academic.

Price JL, Drevets WC (2010) Neurocircuitry of mood disorders. Neuropsychopharmacology 35:192-216.

Radley JJ, Rocher AB, Miller M, Janssen WG, Liston C, Hof PR, McEwen BS, Morrison JH (2006) Repeated stress induces dendritic spine loss in the rat medial prefrontal cortex. Cereb Cortex 16:313-320.

Solomon MB, Herman JP (2009) Sex differences in psychopathology: of gonads, adrenals and mental illness. Physiol Behav 97:250-258.

Sousa N, Lukoyanov NV, Madeira MD, Almeida OF, Paula-Barbosa MM (2000) Reorganization of the morphology of hippocampal neurites and synapses after stress-induced damage correlates with behavioral improvement. Neuroscience 97:253-266.

Swain JE (2008) Baby stimuli and the parent brain: functional neuroimaging of the neural substrates of parent-infant attachment. Psychiatry (Edgmont) 5:28-36.

Verwer RW, Meijer RJ, Van Uum HF, Witter MP (1997) Collateral projections from the rat hippocampal formation to the lateral and medial prefrontal cortex. Hippocampus 7:397-402.

Wellman CL (2001) Dendritic reorganization in pyramidal neurons in medial prefrontal cortex after chronic corticosterone administration. J Neurobiol 49:245-253.

Windle RJ, Kershaw YM, Shanks N, Wood SA, Lightman SL, Ingram CD (2004) Oxytocin attenuates stress-induced c-fos mRNA expression in specific forebrain regions associated with modulation of hypothalamopituitary-adrenal activity. J Neurosci 24:2974-2982. 